Original Article

\title{
2,4-SUBSTITUTED QUINAZOLINE AS JAK2 INHIBITOR: DOCKING AND MOLECULAR DYNAMICS STUDY
}

\author{
SIVA JYOTHI BUGGANA ${ }^{1}$, MANI CHANDRIKA PATURI ${ }^{1}$, RAJENDRA PRASAD VVS ${ }^{*}$
}

${ }^{1}$ Department of Pharmaceutical Chemistry, Bojjam Narasimhulu Pharmacy College for Women, Hyderabad, India, ${ }^{2}$ Centre for Molecular Cancer Research (CMCR), Vishnu Institute of Pharmaceutical and Educational Research, Narsapur, India

Email: rajendraprasad.vvs@viper.ac.in

Received: 07 Nov 2019, Revised and Accepted: 17 Dec 2019

ABSTRACT

Objective: The involvement of Janus kinase2/signal transducer and activator of transcription (JAK2/STAT3) pathway reported in various solid tumors made authors study the conformational changes of JAK2-3e complex which was previously reported with a moderate percentage of In-vitro JAK2 inhibition.

Methods: In this present study Compound 3e was reported with a moderate percentage of inhibition of JAK2 protein selected for performing molecular docking and molecular dynamics studies to elucidate the conformational changes with JAK2-3e complex. Docking studies were performed using ChemSketch to draw the structure of the compound and optimized/energy minimized using the Ligprep module of Schrodinger suite, employing optimized potentials for liquid simulations (OPLS-2005) force field. Molecular dynamics simulations were performed for 10 ns for complex using TIP4PEW water solvent model and neutralized by adding sodium ions.

Results: Docking studies of Compound 3e which has been reported as one of the effective cytotoxic agents and a moderate percentage of In-vitro JAK2 inhibition among the series, showed H-bond interaction with leucine 855, serine936, aspartine994. Dock score and Ligand binding energy with protein suggested compound 3e has shown-4.049,-66.003 kcal/mol respectively. Molecular dynamics simulations elucidated the mechanistic insight of JAK-2 inhibition. The Root means square deviation (RMSD) pattern of both protein and ligands in the JAK2-3e complex observed to be different over 10 ns simulation. In the JAK2-3e complex, an exponential increase in RMSD of C $\alpha$ and side-chain amino acids is observed during the first 1-3 ns simulation and is stabilized till $10 \mathrm{~ns}$. During the $10 \mathrm{~ns}$ simulation, ligand 3e seems to be stable in the complex with an overall deviation $<1 \AA$, despite a drastic increase between 1-3 ns. The ligand RMSD plot suggests that the ligand 3e remained intact within the binding site of the protein and longer time period simulation may elucidate the binding pattern and fate of ligand 3e.

Conclusion: Results from molecular dynamics simulations elucidated the mechanistic insight of JAK-2 inhibition by 2 , 4 disubstituted quinazoline compound that is $\mathrm{N}^{\prime}(2-(4-$ nitrophenyl)quinazoline-4-yl) isonicotinohydrazide) and their binding phenomenon. Molecular docking studies further supported the elucidation of binding patterns of the molecules in the JAK-2 protein environment. Further simulations with a longer time period may provide deeper insights into ligand interactions in the protein environment. It is noteworthy to use compound 3e as a new scaffold for further development of multifunctional compounds

Keywords: Quinazoline, Cytotoxic activity, Molecular docking, JAK2, Molecular dynamics

(C) 2020 The Authors. Published by Innovare Academic Sciences Pvt Ltd. This is an open-access article under the CC BY license (http://creativecommons.org/licenses/by/4.0/A DOI: http://dx.doi.org/10.22159/ijpps.2020v12i2.36306. Journal homepage: https://innovareacademics.in/journals/index.php/ijpps

\section{INTRODUCTION}

Cancer is one of the major threats to human life worldwide and is the second leading cause of death in the United States. An estimated 6, 06,880 Americans will die from cancer in 2019 [1], corresponding to almost 1700 deaths per day. The greatest number of deaths is from cancers of the lung, prostate, and colorectum in men and the lung, breast, and colorectum in women. Even cancer is the second most common cause of death among children aged 1-14 y in the United States. In 2019, an estimated 11,060 children will be diagnosed with cancer and 1,190 will die from the disease.

The sequence of events like cell cycle progression, cell division, and proliferation can be controlled by a cascade of enzymes such as protein kinases [2]. These enzymes are responsible for regulating physiological mechanisms, including cell differentiation, migration, and metabolism. These enzymes are divided into three categories like tyrosine kinases [3], serine/threonine kinases [4], and Histidine kinases [5]. When the protein kinases are expressed in mutated, unregulated forms (or) produced abnormally in high levels these can transform normal cells into carcinogenic phenotypes [6, 7]. JAKSTAT pathway transduces extracellular signals into transcriptional programs which can regulate cell growth and differentiation $[8,9]$. The mammary gland and ovary where the JAK-STAT pathway is extensively investigated. During puberty, pregnancy the mammary cells and ovary cells exposed to signaling pathways that inhibit apoptosis, induce proliferation and invoke differentiation. The signaling pathways are responsible for bringing out these changes in breast and ovarian cells. The distinct functions of STAT3 and STAT5 have received attention in the context of breast cancer and ovarian cancer. STAT3 activity can be modulated through STAT5 activity and their combined functions can have an impact on the progression of breast and ovarian cancers [10]. The cell division and angiogenesis required for tumor growth and metastasis are controlled by protein kinases. Hence there is a necessity to develop non-toxic and selective inhibitors of protein kinases in tumor cells represent the exciting targets for cancer treatment.

The heterocycles are widely known as bioactive molecules and are considered as an important synthetic target for the development of novel therapeutic agents [11-12]. Quinazoline is one of the heterocycles used as a basic framework that produces biologically active compounds and drug molecules [13]. Due to wide range of biological activities like antimicrobial [14, 15], antimalarial [16], anti-inflammatory [17-19] anti-convulsant [20, 21], antihypertensive [22], anti-oxidant [23], anti-viral [24], anti-HIV [25] and anti-cancer [26-29] quinazoline and its derivatives attracted the attention of both biologists and chemists. Quinazoline and its derivatives have been identified as a new class of cancer chemotherapeutic agents [30] with significant therapeutic efficacy against solid tumors [31-43]. 
In our previous work [44] we have evaluated them for their invitro JAK 2 inhibition which we have previously proved their cytotoxic potentials against human breast (MDA-MB-231) and ovarian cancer (SK-O-V3) cell lines. The synthesis of these compounds is shown in scheme [1]. In the present work, the continuation of our previous work in order to elucidate the mechanistic insights of the 2, 4disubstituted quinazoline molecules, we have performed docking studies of compound $3 \mathrm{e}$ against JAK-2 protein. Additionally, molecular dynamics simulations of compound $3 \mathrm{e}$ conducted to elucidate the conformational changes of the JAK-2-Quinazoline complex.

\section{MATERIALS AND METHODS}

\section{Molecular docking and binding energy calculations}

The synthesized quinazoline derivative structure $3 \mathrm{e}$ was drawn using ChemSketch and optimized/energy minimized using the Ligprep module of Schrodinger suite, employing OPLS-2005 (optimized potentials for liquid simulations) force field. A 3D digital structure of the target kinase domain of JAK2 protein was retrieved from the Protein databank website (PDB Id: 3RVG). The protein structure was optimized using the Protein Preparation Wizard, by adding hydrogen atoms to satisfy the valences and optimized by using the OPLS-2005 force field. Protein-ligand interactions and conformational changes were examined by using the Glide XP docking protocol [45]. Glide XP modules include two steps, Receptor Grid generation and ligand docking. A grid was generated considering the volume of the active binding pocket of the protein and all ligands were docked into the active site of the target protein. Glide score includes a steric clash term, adds polar terms featured by
Schrodinger to correct electrostatic mismatches. Glide score is a combination of hydrophilic, hydrophobic, metal-binding groups, Van der Waals energy, freezing rotatable bonds, and polar interactions with receptors. MM/GBSA calculations were performed for the docked protein-ligand complexes to determine the binding energy. The total free energy of binding is then expressed in the form below mentioned equation:

$\Delta \mathrm{G}_{\text {bind }}=\mathrm{G}_{\text {complex }}-\left(\mathrm{G}_{\text {protein }}+\mathrm{G}_{\text {ligand }}\right)$

where $\Delta \mathrm{G}_{\text {bind }}$ is ligand binding energy.

In other terms,

$\Delta \mathrm{G}_{\text {bind }}=\Delta \mathrm{E}_{\text {gas }}+\Delta \mathrm{G}_{\text {solvation }}-\mathrm{T} \Delta \mathrm{S}$

Where gas phase molecular mechanical energy is $\Delta \mathrm{E}_{\text {gas }}$, solvation free energy are $\Delta \mathrm{G}_{\text {solvation }}$ (polar and non-polar contributions) and $\mathrm{T} \Delta \mathrm{S}$ is the entropy [46].

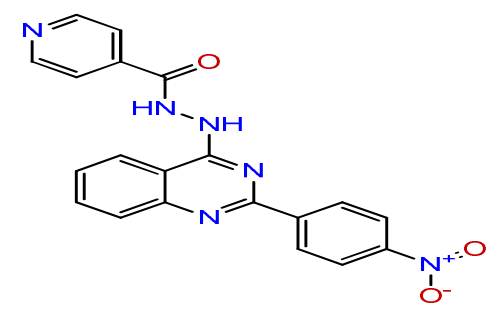

Fig. 1: Compound 3e

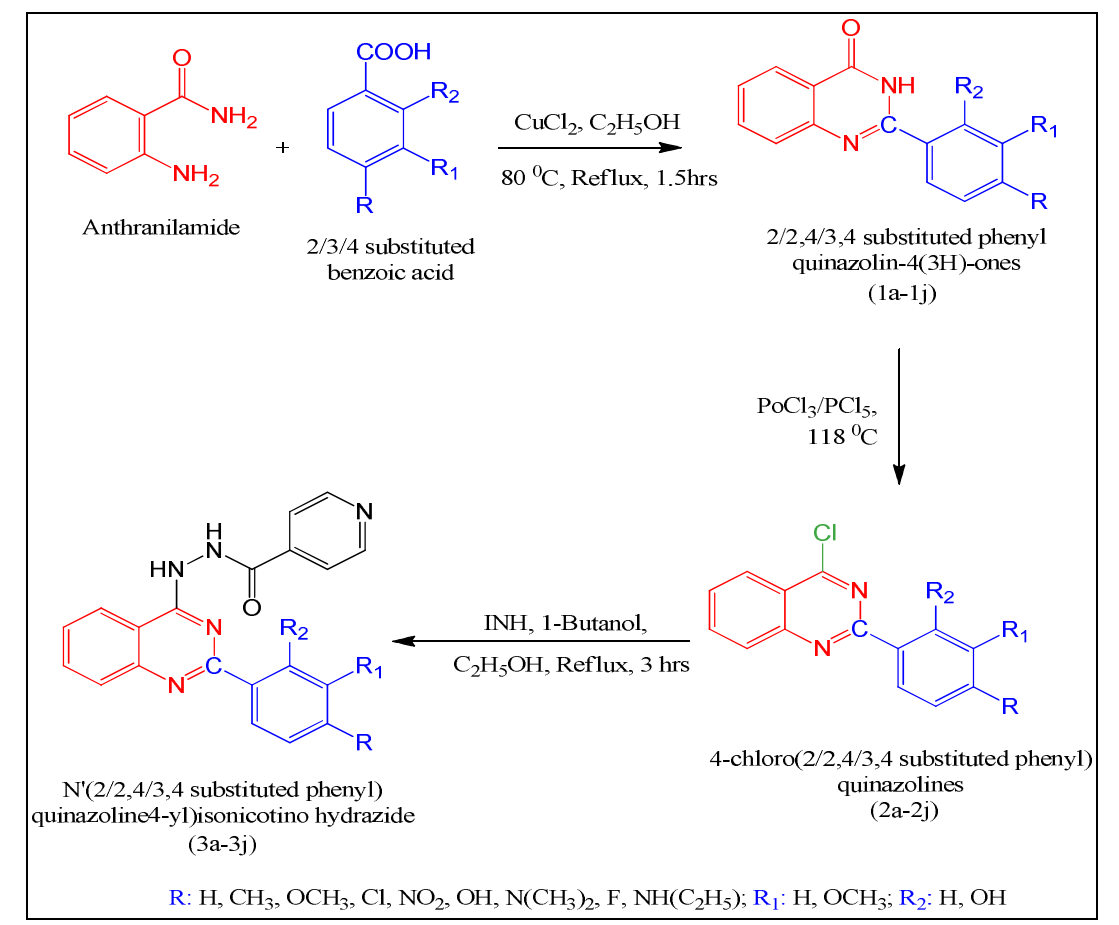

Scheme 1: Synthesis of 2,4-disubstituted quinazolines

\section{Molecular dynamics}

The docked complex of the JAK2 protein and compound 3e was selected for molecular dynamics studies using Desmond by D. E. Shaw Research as discussed in our previous publication [44]. Molecular dynamics simulations were performed for $10 \mathrm{~ns}$ for complex using the TIP4PEW water solvent model and neutralized by adding sodium ions. The system was maintained at $0.15 \mathrm{M}$ salt concentration $\left(\mathrm{Na}^{+} \mathrm{Cl}\right)$ simulating the physiological conditions and dynamics simulations were performed using OPLS 2005 force field and NPT ensemble (constant number of atoms, at constant pressure [1.01352 bar], and constant temperature
[300K] for 10 ns. For system ensemble, Nose-Hoover chain thermostat with a relaxation time of $1 \mathrm{ps}$ and Martyna-Tobias-Klein barostat with 2 ps relaxation time and isocratic cooling style and Coulombic interactions were Cut off at $9 \AA ̊$ radius were used.

\section{RESULTS AND DISCUSSION}

\section{Molecular docking and binding energy calculations}

Considering the association of JAK2 in human solid tumors like breast and ovarian cancers, Molecular docking studies were performed to determine the protein-ligand interactions and also to understand the 
conformational changes in the protein-ligand complex. Docking studies were performed with the compound which was evaluated for in vitro JAK-2 inhibition. Dock score of the tested compound-4.049 (3e). Compound 3e which has been reported as one of the effective cytotoxic agents among the series showed H-bond interaction with leucine 855, serine936, aspartine994 (fig. 2 and 3). Ligand binding energy with protein suggested compound $3 \mathrm{e}$ has shown binding energy of- $66.003 \mathrm{kcal} / \mathrm{mol}$.

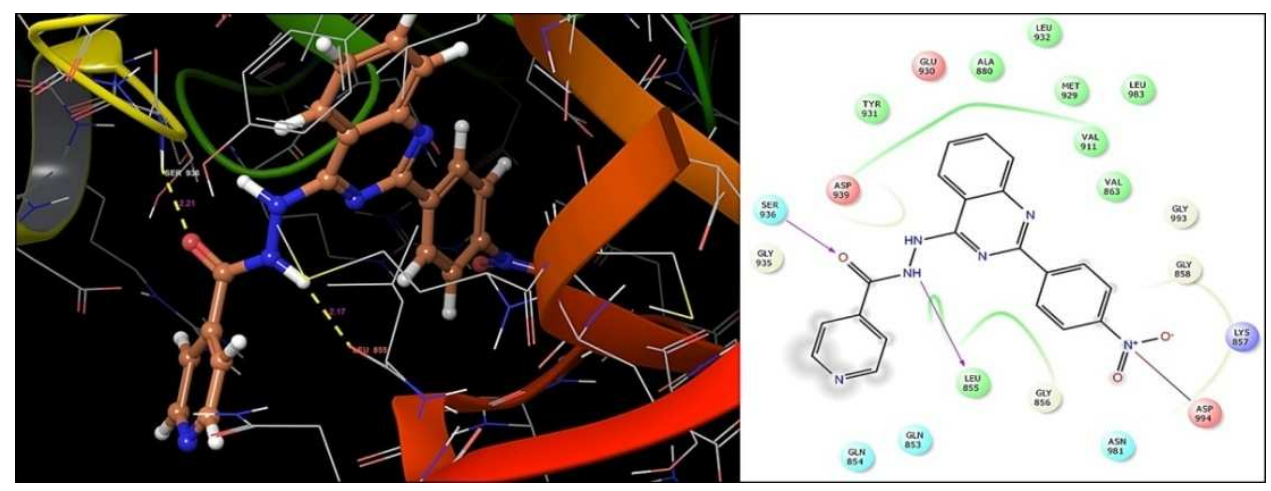

Fig. 2: Protein-ligand interactions of compound 3e with JAK2 protein

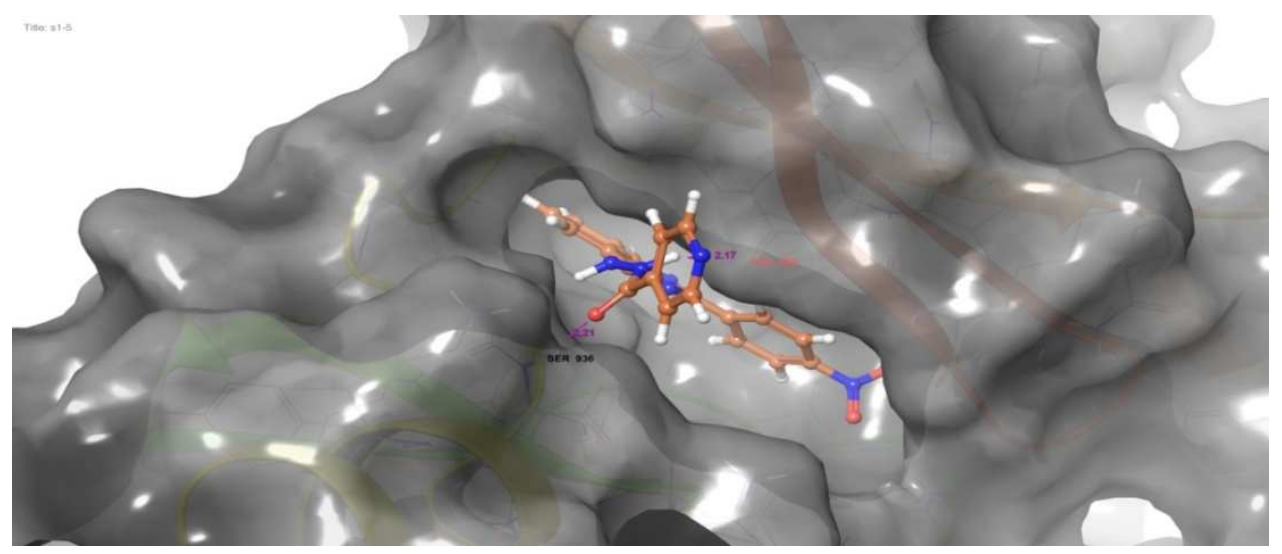

Fig. 3: Protein-ligand interactions of compound 3e with JAK2 protein

\section{Molecular dynamics}

Molecular dynamics simulations performed in the solvent environment at constant temperature and pressure have revealed the conformation changes of the JAK-2-3ecomplex. The stability of the complex is determined in terms of the RMSD and RMSF fig. 4 of the protein components along with the ligand. When compared to the loop region, alpha helices and beta strands are more rigid and thus fluctuations are relatively lower. Overall, the fluctuations in the loop region are observed to be higher in the JAK2-3e complex (up to $5.4 \AA$ ). The RMSD pattern of both protein and ligands in the JAK2-3e complex observed to be different over 10 ns simulation (fig. 5). In the JAK2-3e complex, an exponential increase in RMSD of $\mathrm{C} \alpha$ and side-chain amino acids is observed during the first 1-3 ns simulation and is stabilized till $10 \mathrm{~ns}$. During the $10 \mathrm{~ns}$ simulation, ligand $3 \mathrm{e}$ seems to be stable in the complex with an overall deviation $<1 \AA$, despite a drastic increase between 1-3 ns. The ligand RMSD plot suggests that the ligand 3e remained intact within the binding site of the protein and longer time period simulation may elucidate the binding pattern and fate of ligand $3 \mathrm{e}$.

The ligand-protein contacts between compound 3e and JAK2 during the 10 ns simulation time frame are shown in fig. 6. During the simulation time frame, ASP 939 (46\%), TYR 931 (71\%), and LEU $932(94 \%)$ are the most interacted amino acids with compound 3 e. In compound 3e, a prominent intra-molecular H-bond is observed between the Nitrogen atom of quinazoline moiety and a hydroxyl group. The fate of protein-ligand interactions and change in the total number of contacts over $10 \mathrm{~ns}$ is shown in fig. 7.

In JAK2-3e complex, hydrophilic interactions are predominant in the binding affinity. In JAK2-3e complex, 9 water bridges are observed with GLN 853, LEU932, LYS857, TYR 931, LEU 932, ARG 980, GLY 935, ARG 938 and SER 936 residues. Even ionic interactions also predominant in the binding affinity, two ionic linkages are observed at ARG938, Asp939. The overall interaction diagram of the JAK2-3e complex during molecular dynamic simulations is shown in fig. 8.

The ligand torsion profiles of JAK2-3e seem to be different. The radial plots shown in fig. 9 explains the conformation of the ligand torsion throughout the course of the simulation (beginning of the simulation is in the center and the time evolution is radially outward). In JAK2-3d complex, torsion for the rotatable bonds between pyridine nucleus and carbonyl group, and hydrazide linkage, benzene ring, and nitro group diverge and this could have led to the extended conformational changes of the complex during 13 ns simulation.

The fate of the molecular properties (ligand RMSD, the radius of gyration [rGyr], intramolecular h-bonds, molecular surface area [MolSA], solvent accessible surface area [SASA], and polar surface area [PSA]) of compound 3e determined over $10 \mathrm{~ns}$ and shown in fig. 10. Overall, during 10 ns no large changes were observed in compound 3e ligand properties. 


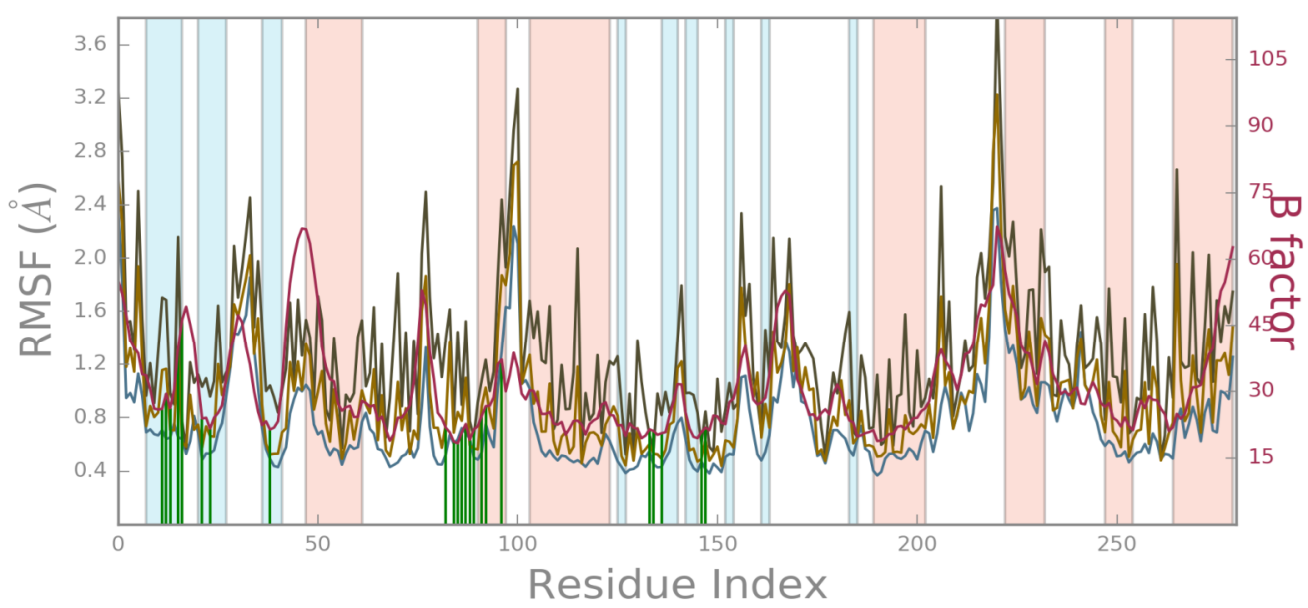

$\mathrm{C} \alpha$ chain $\square$ Heavy Atoms $\square$ B factor

Fig. 4: Root mean square fluctuations (RMSF) for the C-alphas and side-chain residues of JAK2 protein in complex with compound 3e

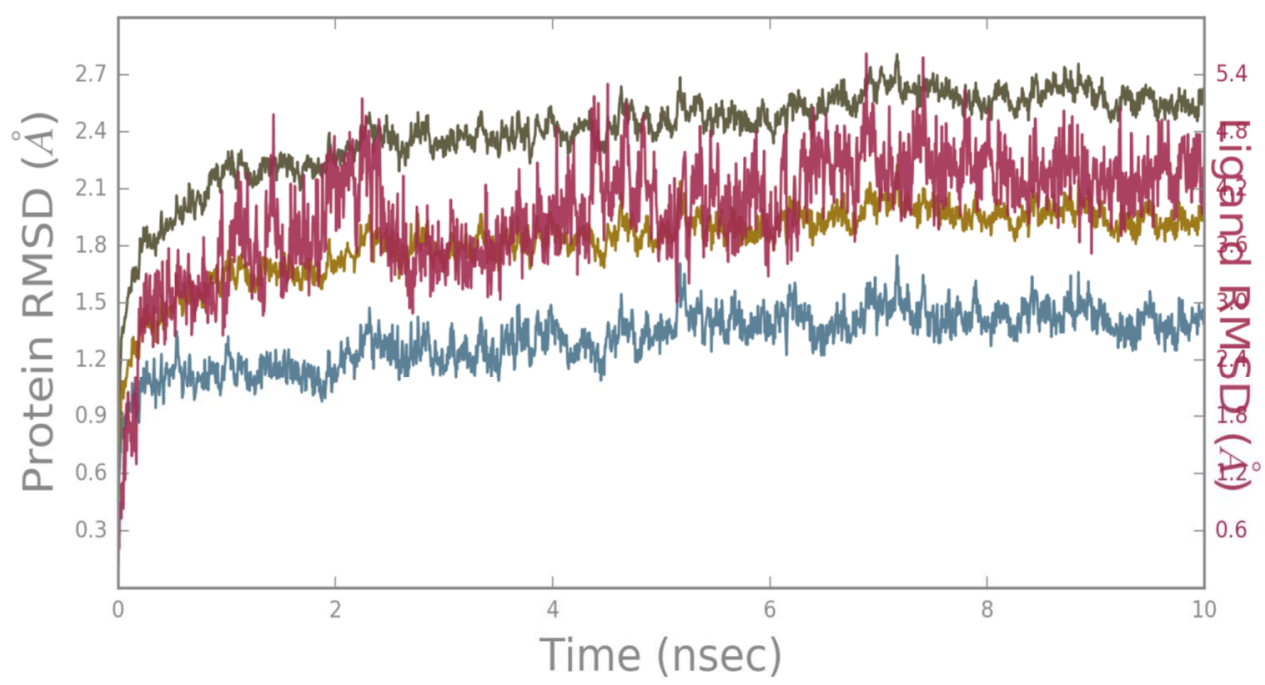

$\square \mathrm{C} \alpha \square$ Side chains $\square$ Heavy atoms $\square$ Lig fit Prot

Fig. 5: Root mean square deviation (RMSD) of simulated complex

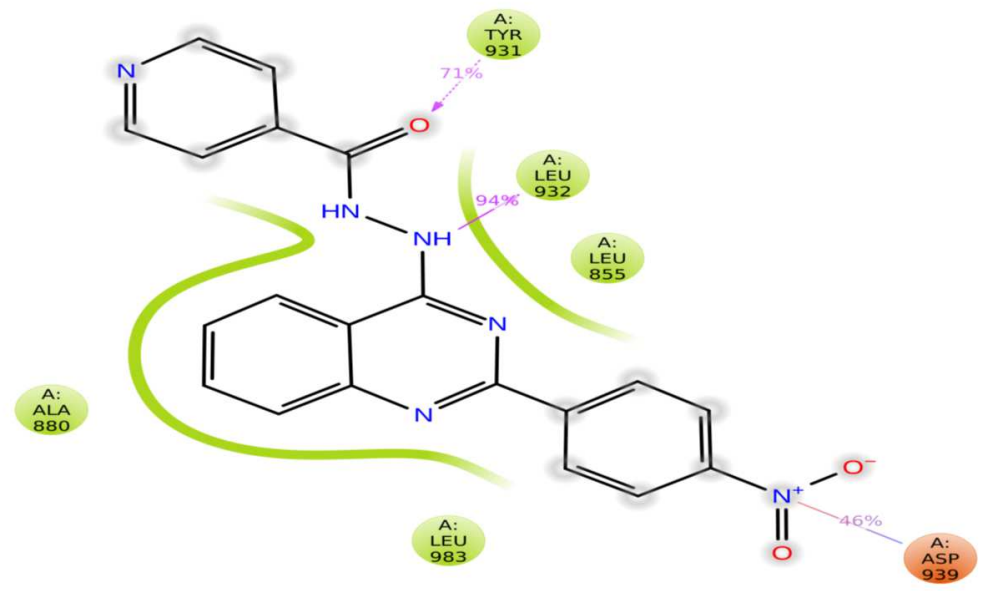

Fig. 6: ligand-protein interaction probabilities in JAK2-3e complex during $10 \mathrm{~ns}$ time period 

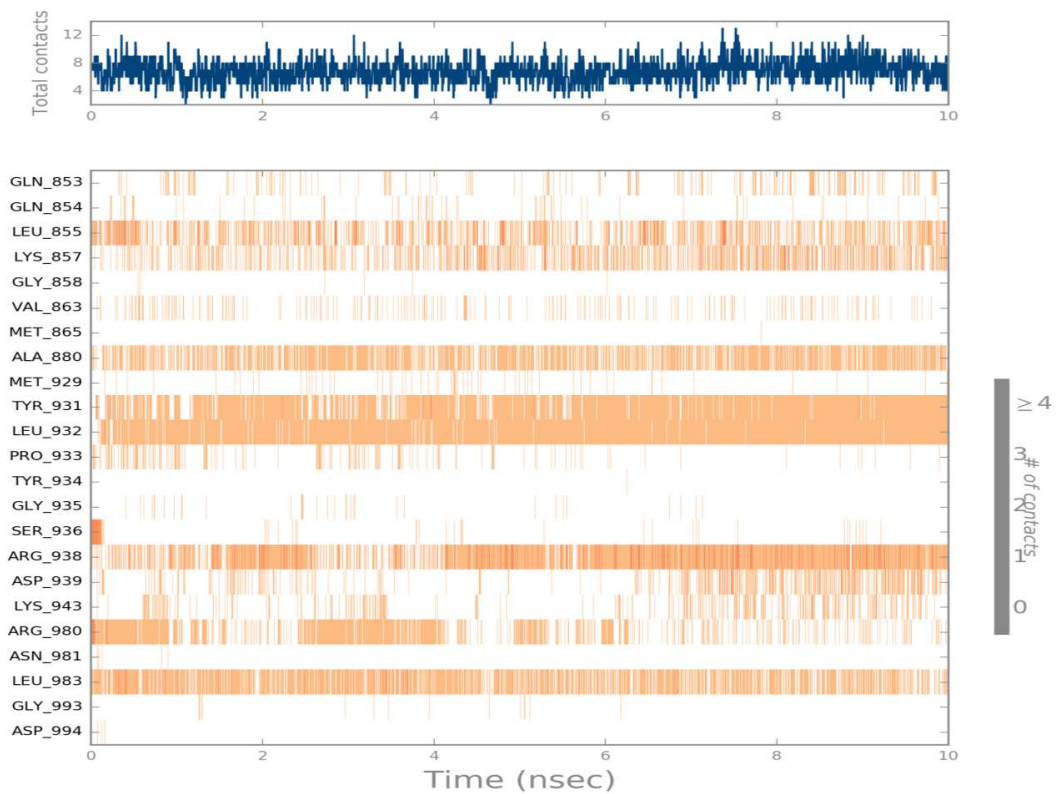

Fig. 7: Protein-ligand interaction diagram for10 ns time period

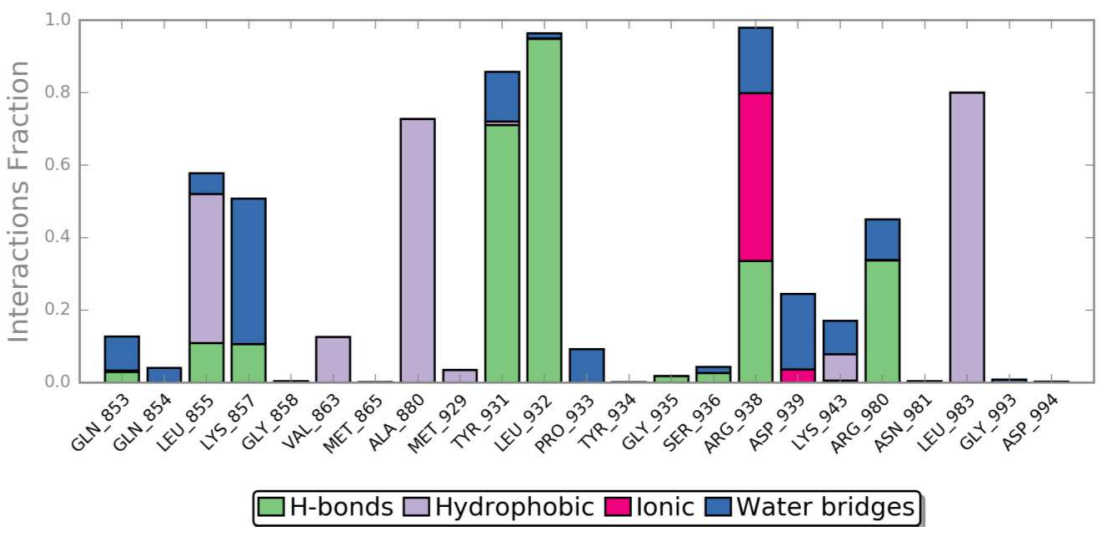

Fig. 8: Interaction diagrams of JAK2-3e complex during molecular dynamic simulations
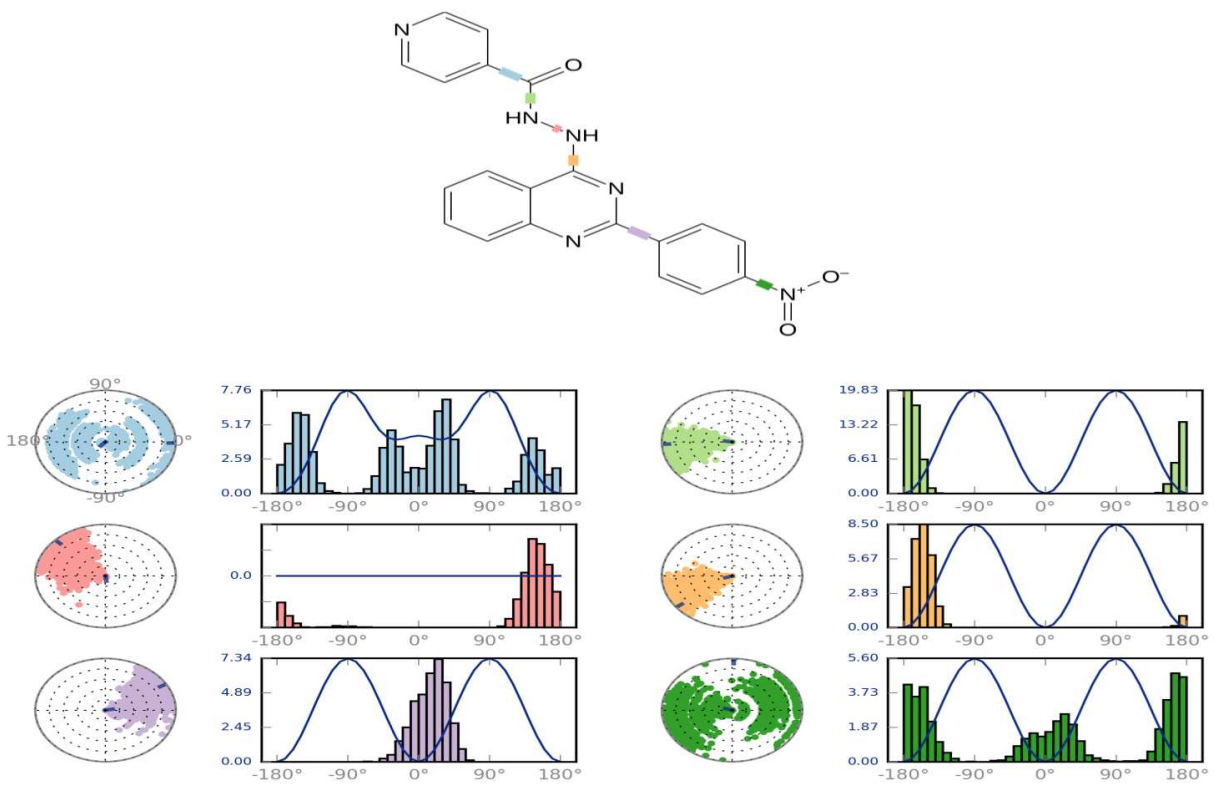

Fig. 9: Ligand torsion profiles of JAK2-3e complex over $10 \mathrm{~ns}$ 


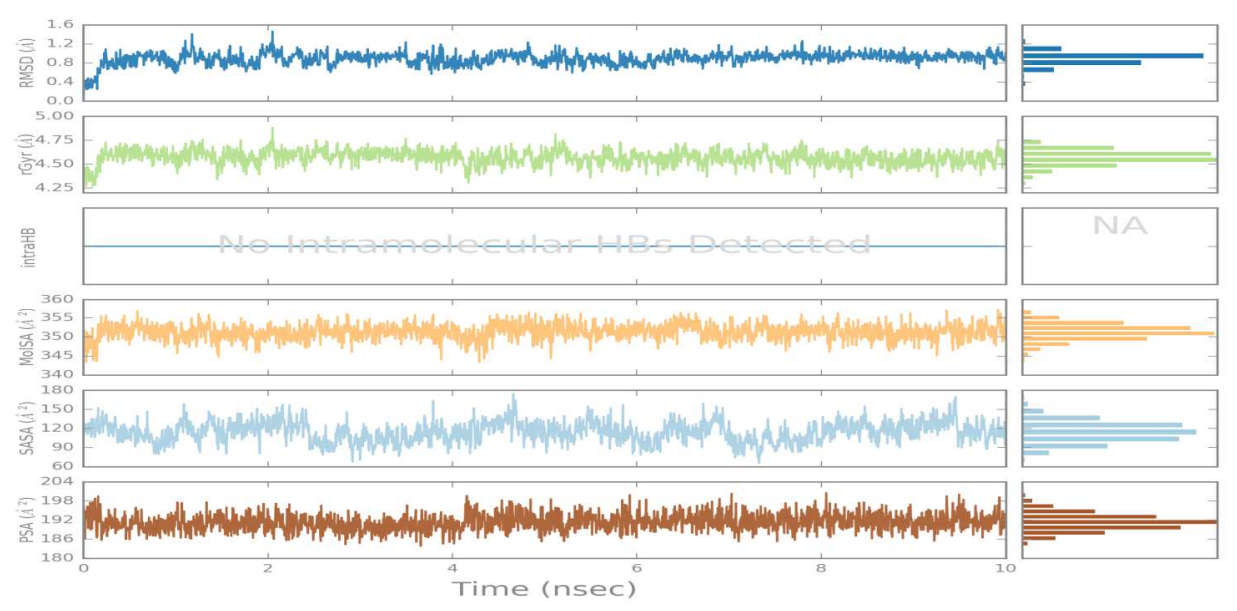

Fig. 10: Fluctuations in the ligand properties over10 ns simulation

\section{DISCUSSION}

We have designed a convenient Insilico method for one of the novel 2, 4 disubstituted quinazoline derivative that is $\mathrm{N}^{\prime}(2-(4-$ nitrophenyl)quinazoline-4-yl) isonicotinohydrazide) which was previously proved for their cytotoxic potentials. Enzyme inhibitory studies suggested that the inhibitory activity of this compound against JAK-2 protein could be the reason for the higher cytotoxicity. When we compare the molecular dynamics study of the compound 3e with our earlier work[44] in the same scheme that is for compound 3b(N1-(2(2,4-dihydroxy phenyl) quinazolin-4-yl) isonicotinohydrazide) and compound 3d(N1-(2(2-phenyl)quinazoline-4-yl)isonicotinohydrazide) for 20ns, it was observed that the compound with dihidroxy substitution shown fluctuation at 5-10ns whereas the compound without substitution shown the fluctuations after $15 \mathrm{~ns}$ only. The compound 3e shown fluctuations during the first 1-3ns only. This may be because of the presence of nitro substitution at the para position. In docking studies, Compound $3 \mathrm{e}$ showed H-bond interaction with leucine 855, serine936, aspartine994, whereas the compounds 3b, 3d showed H-bond interaction with leucine 932, Arginine 938. More hydrogen bond interactions are observed due to nitro substitution at the para position. Hence In this present study Compound 3e from 2,4disubstituted quinazoline was reported with a moderate percentage of inhibition of JAK2 protein selected for performing molecular docking and molecular dynamics studies to elucidate the conformational changes and deeper insights with JAK2-3e complex. From the results, it is noteworthy that the compound $3 e$ can be used as a new scaffold for designing novel cytotoxic agents with fewer toxic effects.

\section{CONCLUSION}

In the current investigation, one of novel 2, 4 disubstituted quinazoline derivatives that are N'(2-(4-nitrophenyl)quinazoline-4yl) isonicotinohydrazide) taken which was previously proved for their cytotoxic potentials. Enzyme inhibitory studies suggested that the inhibitory activity of these compounds against JAK-2 protein could be the reason for the higher cytotoxicity. Molecular docking studies further supported the elucidation of binding patterns of the molecules in the JAK-2 protein environment. Results from molecular dynamics simulations elucidated the mechanistic insight of JAK-2 inhibition by 2, 4 disubstituted quinazoline compound that is $\mathrm{N}^{\prime}(2-$ (4-nitrophenyl)quinazoline-4-yl) isonicotinohydrazide) and their binding phenomenon. Even though simulations are conducted for a shorter duration of the period that is 10 ns gave comparable results with our previous publication. Further simulations with a longer time period may provide deeper insights into ligand interactions in the protein environment. It is noteworthy to use compound $3 \mathrm{e}$ as a new scaffold for further development of multifunctional compounds.

\section{ACKNOWLEDGMENT}

We thank the principals and management of Bojjam Narasimhulu Pharmacy College for Women, Hyderabad, Vishnu Institute of
Pharmaceutical Education and Research, Narsapur, for providing facilities to carry out the work.

\section{FUNDING}

Nil

\section{AUTHORS CONTRIBUTIONS}

Siva Jyothi Buggana contributed to the preparation of the manuscript, Dr. Mani Chandrika Paturi supervised the manuscript preparation and Dr. V. V. S. Rajendra Prasad organized and reviewed the manuscript.

\section{CONFLICT OF INTERESTS}

The authors declare no conflicts

\section{REFERENCES}

1. Rebecca L Siegel et al. Cancer statistics. CA Cancer J Clinicians 2019;69:7-34.

2. Rania SM Ismail. Recent advances in 4-Amino quinazoline based scaffold derivatives targeting EGFR kinases as anticancer agents. Future J Pharm Sci 2016:2:9-19.

3. Jatav V, Kashaw S, Mishra P. Synthesis and antimicrobial activity of some new 3-[5-(4-substituted) phenyl-1,3,4oxadiazole-2yl]-2-styrylquinazoline-4(3H)-ones. Med Chem Res 2008:17:205-11.

4. Leena KP, Subin MZ, Deepthy CH. In silico design, synthesis and characterization of some novel benzothiazole derivatives as anticancer agents. Asian J Pharm Clin Res 2017;10:500-15.

5. Priya D, Srimathi R, Anjana GV. Synthesis and evaluation of some mannich bases of the quinazolinone nucleus. Asian J Pharm Clin Res 2018;11:407-9.

6. Chandregowda V, Kush AK, Chandrasekara Reddy G. Synthesis and in vitroantitumor activities of novel 4-anilinoquinazoline derivatives. Eur J Med Chem 2009;44:3046-55.

7. Al-Rashood ST, Aboldahab IA, Nagi MN, Abouzeid LA, AbdelAziz AA, Abdel-Hamide SG, et al. Synthesis, dihydrofolate reductase inhibition, antitumor testing, and molecular modeling study of some new $4(3 \mathrm{H})$-quinazolinone analogs. Bioorg Med Chem 2006;14:8608-21.

8. Oshealji J. The JAK-STAT pathway, impact on human disease and therapeutic invention. Annu Rou Med 2015;66:311-28.

9. Liongue C, Sertori R, Ward AC. Evolution of cytokine receptor signaling. J Immunol 2016;197:11-8.

10. George Speyer Hans. Jak stat signaling and cancer; opportunities, benefits and side effects of targeted inhibition. Mol Cell Endocrinol 2017;45:1-14.

11. Shagufta. An insight therapeutic potential of quinazoline derivatives as anticancer agents. Med Chem Commun 2017;8:1-26.

12. Ahmad I, Shagufta. Recent developments in steroidal and nonsteroidal aromatase inhibitors for the chemoprevention of 
estrogen-dependent breast cancer. Eur J Med Chem 2015;102:375-86.

13. Ahmad I, Shagufta. Sulfones: an important class of organic compounds with diverse biological activities. Int J Pharm Sci 2015;7:19-27

14. Demeunynck M, Baussanne I. Survey of recent literature related to the biologically active $4(3 \mathrm{H})$-quinazolinones containing fused heterocycles. Curr Med Chem 2013;20:794-814.

15. Raghavendra NM, Thampi P, Guru Basavaraja Swami PM, Sriram D. Synthesis and antimicrobial activities of some novel substituted 2-imidazolyl-N-(4-oxo-quinazolin-3(4H)-yl)acetamides. Chem Pharm Bull 2007;55:1615-9.

16. Paneersalvam P, Rather BA, Reddy DRS, Kumar NR. Synthesis and anti-microbial screening of some Schiff bases of 3-amino6,8-dibromo-2-phenylquinazolin-4(3H)-ones. Eur J Med Chem 2009;44:2328-33.

17. Verhaeghe P, Azas N, Gasquet M, Hutter S, Ducros C, Laget M, et al. Synthesis and antiplasmodial activity of new 4-aryl-2trichloromethylquinazolines. Bioorg Med Chem Lett 2008;18:396-401.

18. Saravanan G, Paneersalvam P, Prakash CR. Synthesis, analgesic and anti-inflammatory screening of novel Schiff bases of 3amino-2-methyl quinazoline 4-(3H)-one. Der Pharm Lett 2010;2:216-26.

19. Alagarsamy V, Solomon VR, Sheorey RV, Jayakumar R. 3-(3Ethylphenyl)-2-substitutedhydrazino-3H-quinazolin-4-one derivatives: new class of analgesic and anti-inflammatory agents. Chem Biol Drug Des 2009;73:471-9.

20. Smits RA, Adami M, Istyastono EP, Zuiderveld OP, van Dam CME. Synthesis and QSAR of quinazoline sulfonamide as highly potent human histamine $\mathrm{H} 4$ receptor inverse agonists. J Med Chem 2010;53:2390-400.

21. Georgey H, Abdel Gawad N, Abbas S. Synthesis and anticonvulsant activity of some quinazoline-4-(3H)-one derivatives. Molecules 2008;13:2557-69.

22. Patel NB. Synthesis and microbial studies of (4-oxothiazolidinyl) sulfonamides bearing quinazolin-4(3H) ones. Acta Polo Pharm Drug Res 2010;67:267-75.

23. Ismail MAH, Barker S, Abau El Ella DA, Abouzid KAM, Toubar RA. Design and synthesis of new tetrazolyl-and carboxybiphenylylmethyl-quinazolin-4-one derivatives as angiotensin II AT1 receptor antagonists. J Med Chem 2006;49:1526-35.

24. Zaranappa, Vagdevi HM, Lokesh MR, Gowdarshivannanava BC. Synthesis and antioxidant activity of 3-substituted schiff bases of quinazoline-2,4-diones. Int J Chem Tech Res 2012;4:1527-33.

25. Krishnan SK. Synthesis, antiviral and cytotoxic investigation of 2-phenyl-3-substituted quinazolin-4(3H)-ones. Eur Rev Med Pharm Sci 2011;15:673-81.

26. Pati B, Banerjee S. Quinazolines: an illustrated review. J Adv Pharm Edu Res 2013;3:136-51.

27. Katrin SN. Chemotherapy and dietary phytochemical agents. Chemother Res Prac 2012;3:22-7.

28. Manasa AK, Sidhaye RV, Radhika G, Nalini CN. Synthesis, antioxidant and anticancer activity of quinazoline derivatives. Curr Pharma Res 2011;1:101-5.

29. Nerkar B, Saxena A, Ghone S, Thakeri AK. In silico screening, synthesis and in vitro evaluation of some quinazolinone and pyridine derivatives as dihydrofolate reductase inhibitors for anticancer activity. E-J Chem 2009;6:97-102.

30. Ahmed MF, Youns M. Synthesis and biological evaluation of a novel series of 6, 8-Dibromo-4 $(3 H)$ quinazolinone derivatives as anticancer agents. Archiv Der Pharmazie 2013;346:610-7.

31. Moon DO, Kim MO, Heo MS, Lee JD, Choi YH, Kim GY. Gefitinib induces apoptosis and decreases telomerase activity in MDA-
MB-231 human breast cancer cells. Arch Pharm Res 2009;32:1351-60.

32. Sirisoma N, Pervin A, Zhang H, Jiang S, Adam Willardsen J. Discovery of N-methyl-4-(4-methoxyanilino)quinazolines as potent apoptosis inducers. Structure-activity relationship of the quinazoline ring. Bioorg Med Chem Lett 2010;20:2330-4.

33. Font M, Gonzalez A, Palop JA, Sanmartin C. New insights into the structural requirements for pro-apoptotic agents based on 2,4-diaminoquinazoline, 2,4-diaminopyrido[2,3-d]pyrimidine and 2,4-diaminopyrimidine derivatives. Eur J Med Chem 2011;46:3887-99.

34. Liu F, Lovejoy DB, Hassani AA, He Y, Herold JM, Chen X, et al. Optimization of cellular activity of G9aInhibitors 7aminoalkoxy-quinazolines. J Med Chem 2011;54:6139-50.

35. EI-Azab AS, Al-Omar MA, Abdel Aziz AA Metal. Design, synthesis and biological evaluation of novel quinazoline derivatives as potential antitumor agents: molecular docking study. Eur J Med Chem 2010;45:4188-98.

36. Al-Obaid AM, Abdel-Hamide SGA, El-Kashef HA. Substituted quinazolines, part 3. Synthesis, in vitro antitumor activity and molecular modeling study of certain 2-thieno-4(3H)quinazolinone analogs. Eur J Med Chem 2009;44:2379-91.

37. Alafeefy AM, Kadi AA, El-Azab AS, Abdel-Hamide SG, Daba MH. Synthesis, analgesic and anti-inflammatory evaluation of some new 3H-quinazolin-4-one derivatives. Archiv Der Pharmazie 2008;341:377-85.

38. Kumar A, Sharma S, Archana A, Bajaj K, Sharma Setal. Some new 2,3,6-trisubstituted quinazolinones as a potent antiinflammatory, analgesic and COX-II inhibitors. Bioorg Med Chem 2003;11:5293-9.

39. El-Azab AS, Kamal EH. Design and synthesis of novel 7aminoquinazoline derivatives: antitumor and anticonvulsant activities. Bioorg Med Chem Lett 2012;22:1879-85.

40. Kashaw SK, Kashaw V, Mishra P, Jain NK, Stables JP. Synthesis, anticonvulsant and CNS depressant activity of some new bioactive 1-(4-substituted-phenyl)-3-(4-oxo-2-phenyl/ethyl4H-quinazolin-3-yl)-urea. Eur J Med Chem 2009;44:4335-43.

41. Archana V, Srivastava K, Kumar A. Synthesis of some newer derivatives of substitutedquinazolinonyl-2-oxo/thiobarbituric acid as potent anticonvulsant agents. Bioorg Med Chem 2004;12:1257-64.

42. Al-Suwaidan IA, Alanazi AM, Abdel-Aziz AA, Mohamed MA, ElAzab AS. Design, synthesis and biological evaluation of 2mercapto-3-phenethylquinazoline bearing anilide fragments as potential antitumor agents: molecular docking study. Bioorg Med Chem Lett 2013;23:3935-41.

43. Abdel Gawad NM, Georgey HH, Youssef RM, El-Sayed NA. Synthesis and antitumor activity of some 2, 3-disubstituted quinazolin-4(3H)-ones and 4, 6-disubstituted-1, 2, 3, 4tetrahydro quinazoline-2H-ones. Eur J Med Chem 2010;45:6058-67.

44. B Siva Jyothi, MC Paturi, H Perka, DR Gade, VVS Rajendra Prasad. Novel 2,4-disubstituted quinazolines as cytotoxic agents and JAK2 inhibitors: synthesis, in vitro evaluation and molecular dynamics studies. Comput Biol Chem 2019;79:110-8.

45. VVS Rajendra Prasad, DR Gade, D Appaji, GJ Peters, YC Mayur. Chemosensitizing acridones: in vitro calmodulin-dependent cAMP phosphodiesterase inhibition, docking, pharmacophore modeling, and 3D QSAR studies. J Mol Graph Model 2013;40:116-24.

46. VVS Rajendra Prasad, DR Gade, I Kathmann, M Amareswararao, GJ Peters. Nitric oxide-releasing acridone carboxamide derivatives as reverters of doxorubicin resistance in MCF7/Dx cancer cells. Bioorg Chem 2016;64:51-8. 